\title{
Estudio de los factores de riesgo para el cancer de mama en mujeres uruguayas
}

DOI: $10.46981 / \mathrm{sfjhv2n1-012}$

Received in: November 1st, 2020

Accepted in: December 30th, 2020

\author{
Natalia Camejo \\ Prof. Adj. del Servicio de Oncología Clínica, Hospital de Clínicas, Facultad de Medicina, Universidad \\ de la República. Uruguay. \\ Servicio de Oncología Clínica. Hospital de Clínicas \\ Avda. Italia s/n. CP 11600. Montevideo, Uruguay \\ Correo electrónico: ncam3@yahoo.com
}

\section{Cecilia Castillo}

Ex Prof. Adj. del Servicio de Oncología Clínica, Hospital de Clínicas, Facultad de Medicina, Universidad de la República. Uruguay.

\section{Nora Artagaveytia}

Prof. Adj. del Departamento Básico de Medicina, Hospital de Clínicas, Facultad de Medicina, Universidad de la República. Uruguay.

\section{Ana Laura Hernandez}

Prof. Agda. del Departamento de Psicologia Medica, Hospital de Clínicas, Facultad de Medicina, Universidad de la República. Uruguay.

\section{Andrea Schiavone}

Ex Asistente del Servicio de Oncología Clínica, Hospital de Clínicas, Facultad de Medicina, Universidad de la República, Uruguay.

\section{Milans Soledad}

Prof. Adj.con Orientación en Imagenología Mamaria del Departamento de Imagenología, Hospital de Clínicas, Facultad de Medicina, Universidad de la República, Uruguay.

\section{Guillermo Laviña}

Ex Prof. Adj. Clinica Quirúrgica 1, Facultad de Medicina, Universidad de la República. Uruguay.

\section{Lucía Delgado}

Ex Prof. Directora del Servicio de Oncología Clínica, Hospital de Clínicas, Facultad de Medicina, Universidad de la República. Uruguay.

\section{RESUMEN}

En Uruguay el cáncer de mama ocupa el primer lugar en incidencia y mortalidad por cáncer en la mujer. El conocimiento de la distribución de los factores de riesgo relacionados con el desarrollo de la enfermedad facilita la incorporación de estrategias de prevención en la práctica clínica habitual, lo que podría contribuir a disminuir los índices de morbimortalidad. Objetivos: conocer el perfil epidemiológico para el cáncer de mama y la frecuencia del control mamográfico en la población encuestada.- Material y Métodos: Se aplicó una encuesta a mujeres uruguayas. Luego de realizada la misma, se les brindó información sobre los factores de riesgo y protectores y sobre la importancia de realizar el control mamográfico. Resultados: Se incluyeron 296 encuestadas, la mediana de edad fue 36 
años (18-80 años). Excluyendo el sexo femenino (factor de riesgo presente en todas las encuestadas), 133 encuestadas $(44,9 \%)$ presentaban uno o más factores de riego. La mayoría de las mujeres mayores de 50 años $(117,90 \%)$, realizaban control mamográfico al menos en forma bianual. Conclusiónes: Concordantemente con lo reportado por el Programa Nacional de Cáncer de Mama, 44,9\% de las encuestadas tuvieron algún factor de riesgo. La mayoría de las encuestadas mayores de 50 años realizaban control mamográfico y clínico al menos en forma bianual. Si bien, debido a las características del trabajo, solamente podemos sacar conclusiones sobre las mujeres incluidas, los datos recogidos nos permiten conocer mejor el perfil epidemiológico de la población uruguaya, lo que puede contribuir a las prácticas de prevención.

Palabras clave: cáncer de mama, factores de riesgo, prevención.

\section{INTRODUCCIÓN}

El cáncer es un problema de significativa importancia en el tablero epidemiológico del Uruguay. De hecho, tomando en cuenta ambos sexos en forma conjunta, el cáncer es la segunda causa de muerte, después de las enfermedades cardiovasculares, siendo causante de casi un cuarto $(24,6 \%)$ del total de los fallecimientos registrados en nuestro país cada año. Se registran anualmente casi 16.179 casos nuevos y mueren más de 7.995 uruguayos por esta causa $(1,2)$.

Al igual de lo que se observa a nivel mundial, el cáncer de mama $(\mathrm{CM})$ en Uruguay es el más frecuente en el sexo femenino y también la principal causa de muerte por cáncer. Cada año se registran unos 1.926 casos nuevos y unas 670 mujeres mueren como consecuencia de esta enfermedad. En Uruguay, la probablidad de padecer CM a lo largo de la vida es de una cada 11 mujeres. La edad mediana de las pacientes a las que se les diagnosticó su primer CM infiltrante en el período 2012-2016 fue 63 años (51-74 años). La gran mayoría $(69,5 \%)$ de los casos se diagnostican en estadios tempranos reafirmando la importancia de la prevención secundaria en esta patología $(3,4)$.

Si bien en los últimos años se ha comprobado una disminución de la mortalidad por esta causa, lo que podría estar vinculado a un incremento de la detección temprana, una mejora en los tratamientos y en la integración de equipos interdisciplinarios en esta patología (2), el CM continúa generando una gran carga, tanto a nivel social como económico, por el impacto que produce en la persona afectada y en su entorno. La carga económica para la sociedad se configura mediante costos directos e indirectos. A título de ejemplo, dentro de los costos directos se encuentran los de atención médica y, dentro de los costos indirectos, deben tenerse en cuenta el ausentismo laboral y las pensiones por invalidez, viudez y orfandad. Por estas razones enunciadas, entre otras, es importante desarrollar políticas y programas para la prevención y el control de tales enfermedades.

La prevención del CM puede desarrollarse en diferentes niveles. La prevención primaria tiene como objetivo evitar que el individuo desarrolle la enfermedad, y se basa en el conocimiento de los 
factores de riesgo y de los factores protectores. La prevención secundaria tiene como objetivo lograr la curación de la enfermedad y se centra en la detección precoz y el tratamiento oportuno, en etapas donde la curabilidad es alta, con secuelas mínimas o directamente sin ellas (5).

\section{PREVENCIÓN PRIMARIA EN EL CM}

La prevención primaria del CM está orientada a la modificación de los factores de riesgo pasibles de cambio y a fomentar los factores protectores. Desarrollar esta clase de prevención en la población general es complejo actualmente ya que, en la mayoría de las personas que desarrollan la enfermedad, únicamente se reconocen dos factores de riesgo y ellos no son modificables: el sexo femenino y la edad.

\section{FACTORES DE RIESGO}

Si bien la etiología del CM, al igual que acontece en la mayoría de los cánceres, es desconocida, se reconocen diversos factores de riesgo involucrados en su génesis. Estos se pueden clasificar en: modificables y no modificables (tabla 1). Conocer la distribución de los factores de riesgo es esencial para efectuar intervenciones de promoción de salud en la comunidad, con el fin de promover el bienestar y la salud de las mujeres.

Aproximadamente en la mitad de las pacientes diagnosticadas de CM se detecta algún factor de riesgo conocido, diferente al sexo femenino y la edad. Entre de los factores de riesgo es de vital interés reconocer aquellos que son modificables, entre los cuales se destacan el sobrepeso u obesidad, consumo nocivo de bebidas alcohólicas y uso de terapia de reemplazo hormonal. Además, existen factores protectores como la práctica regular de ejercicio (6). El entendimiento de estos factores nos permite planificar acciones de prevención primaria en nuestro entorno acordes con las características de la población destinataria.

\section{PREVENCIÓN SECUNDARIA}

Las tasas de mortalidad por CM han descendido en los últimos años, debido al aumento de la detección temprana mediante el uso de mamografía de tamizaje. Hasta el momento la mamografía constituye el mejor método para el diagnóstico oportuno y está demostrado que es efectiva a fin de reducir la mortalidad por esta enfermedad (7). De acuerdo a las Guías de Práctica Clínica de Detección Temprana del Cáncer de Mama del Ministerio de Salud Pública (MSP), se recomienda realizar tamizaje mamográfico sistemático cada dos años a las mujeres con edad comprendida entre 50-69 años, intervalo etario en el cual el beneficio del tamizaje con mamografía es mayor y los riesgos (sobrediagnóstico, falsos positivos, etc) menores. En el caso de las mujeres con edades comprendidas entre 40-49 años y 
70-74 años, se sugiere individualizar, analizando con la paciente los potenciales beneficios y riesgos de realizar dicho estudio.

\subsection{OBJETIVO:}

Conocer el perfil epidemiológico para el CM y la frecuencia del control clínico y mamográfico en la población encuestada

\subsection{OBJETIVOS ESPECÍFICOS:}

- Conocer la prevalencia de los principales factores de riesgo para CM en la población encuestada.

- Medir la frecuencia con que se realiza el control clínico y el estudio mamográfico.

- Comparar los resultados con las bases de datos a nivel nacional.

\section{MATERIAL Y MÉTODOS}

Se trata de un estudio observacional y prospectivo, en el que se incluyeron las mujeres que concurrieron a las jornadas de prevención del CM realizadas en conjunto por la Comisión Honoraria de Lucha contra el Cáncer (CHLC) y el Servicio de Oncología Clínica del Hospital de Clínicas desde el 2012 hasta el 2019 inclusive. El cuestionario fue respondido de forma individual por cada participante y de manera anónima.

La encuesta fue respondida de forma individual por cada participante y de manera anónima, dándole tiempo suficiente para responder a todas las preguntas. Dicha encuesta fue diseñada ad hoc para indagar sobre los principales factores de riesgo para desarrollar la enfermedad, la realización del examen mamario y mamografía de screening, en forma comprensible (anexo 1). Consistió en 12 preguntas la mayoría cerradas, ya fueren dicotómicas o con múltiples alternativas. Las mismas estaban destinadas a conocer el comportamiento de la población en relación a los factores de riesgo para el CM y medir la frecuencia con que se realiza el examen mamario y el estudio mamográfico.

Las variables incluidas fueron: antecedentes familiares de CM; antecedentes personales de cáncer y enfermedades proliferativas de la mama; elevada densidad mamaria; factores hormonales; consumo de alcohol; y ejercicio físico.

Los datos fueron recopilados, analizados y comparados con los datos uruguayos disponibles. Los mismos fueron procesados en una hoja de cálculo (Microsoft Excel) y como medidas de resumen se utilizaron las frecuencias relativas y absolutas.

El estudio propuesto se realizó de acuerdo con las normas éticas internacionales para las investigaciones biomédicas: "Normas del MERCOSUR sobre regulación de los estudios clínicos” y la 
"Declaración de Helsinski”, y con el reglamento de investigación aprobado por la Comisión Nacional de Ética en 2019.

\section{RESULTADOS}

Se incluyeron 296 encuestadas, 91 procedentes de Montevideo, 37 de Paysandú, 18 de Rocha, 36 de Tacuarembó, 35 de Maldonado y 79 de Soriano.

La integridad de las personas encuestadas fue de sexo femenino, y la mediana de edad fue 36 años (18-80 años).

Descartando como factor de riesgo el sexo femenino (pues se halla presente en todas las encuestadas), 133 encuestadas (44,9\%) presentaban uno o más factores de riesgo para CM. Treinta y siete encuestadas tenían un sólo factor de riesgo, 30 (10 \%) tenían dos factores, y únicamente 8 (2,7\%) de las encuestadas tuvieron tres o más factores de riesgo.

La mayor parte de las encuestadas (192, 64,8 \%) fueron premenopáusicas, 102 (34,4\%) posmenopáusicas y el resto ( 2 encuestadas) perimenopáusicas.

Ciento ochenta encuestadas $(60,8 \%)$ tenían hijos, de éstas ciento cuarenta y cuatro (80 \%) tuvieron hijos antes de los 30 años, y la mayoría (151 pacientes, o sea el 83,8\%) dieron de mamar durante más de 6 meses.

Treinta y seis encuestadas $(12,1 \%)$ tenían antecedentes personales de CM, y veintisiete de ellas (75\%) desarrolló la enfermedad luego de los 50 años.

Setenta y siete encuestadas (26\%) tenían antecedentes familiares de cáncer, 20 (7\%) tenían dos o más familiares afectados, 18 (6,1\%) tenían un familiar de primer grado afectado, y $11(3,7 \%)$ tenían dos o más familiares afectados y uno de estos era de primer grado.

Entre las encuestadas posmenopáusicas, 67 (65,6\%) tuvieron su menopausia entre los 45 y 55 años, 21 (20,5\%) tuvieron menopausia tardía (luego de los 55 años) y 14 (13,7\%) temprana (antes de los 45 años). La mayoría (92 encuestadas, 90,1\%) no recibió THR, el resto sí la recibió. De las 10 encuestadas que recibieron THR, 3 recibieron THR combinada, 2 con progestágenos y en 5 casos no se contaba con el dato.

Ciento noventa y siete encuestadas $(66,5 \%)$ realizaban ejercicio en forma regular, la mayor parte (138 o sea el 70\%) caminaba al menos 30 minutos 3 veces a la semana, y las demás realizaban otras formas de ejercicio (28 bicicleta, 15 yoga, 5 taichi, 5 aparatos, 2 gimnasia, 2 pileta y 2 pilates). El resto de las encuestadas (34\%) eran sedentarias.

Más de la mitad (180 encuestadas, 60,8\%) no consumía alcohol. Del total de consumidoras de alcohol, $104(89,6 \%)$ consumían menos de tres copas a la semana. 
La mayoría de las mujeres mayores de 50 años (117, 90\%), realizan control mamográfico y clínico al menos en forma bianual; el resto no realizan control mamográfico ni examen mamario en forma regular.

\section{DISCUSIÓN}

Como fue mencionado previamente la etiología del CM es desconocida, si bien múltiples estudios indagan sobre factores de riesgo que permitan predecir y cuantificar el riesgo de desarrollar la enfermedad, la mayoría de las veces se trata de un CM esporádico, no siendo posible identificar factores de riesgo implicados en su génesis más allá del sexo y la edad

De las 296 encuestadas, 133 (que equivalen al 44,9\%) presentaban uno o más factores de riesgo para CM. Esta frecuencia es similar a la identificada a nivel nacional por el Programa Nacional de Cáncer de Mama (PRONACAM), que fue del $43 \%$ (8) y a la identificada previamente por nuestro equipo de trabajo (40\%) (9).

De la totalidad de las encuestadas, 77 (26\%) presentaban antecedentes familiares; sin embargo, únicamente 18 encuestadas $(6,1 \%)$ tenían un familiar de primer grado afectado. Si bien esta proporción es similar a la evidenciada por nuestro equipo de trabajo en un estudio previo $(7,6 \%)(9)$; es inferior al porcentaje que emerge de la evaluación efectuada por el PRONACAM $(13,6 \%)(8)$.

La diferencia podría explicarse porque la población analizada en nuestro estudio es una población más joven, con una mediana de 36 años en el presente estudio y de 34 años en el estudio publicado previamente, es decir se trata de mujeres jóvenes cuyos familiares de primer grado quizá no han alcanzado aún la edad de los picos de incidencia del CM. Entretanto, la población evaluada por el PRONACAM incluye mujeres con una mediana de 52 años de edad (8).

Con respecto a los factores protectores, la mayor parte de las encuestadas (197 encuestadas, $66,5 \%$ ) realizaban ejercicio, y únicamente el 33,3\% era sedentaria. Este porcentaje de sedentarismo es inferior al evidenciado en la Primera Encuesta Nacional de Factores de Riesgo de Enfermedades Crónicas No Transmisibles (ECNT), en la cual se reporta un 40,7\% de personas sedentarias dentro del sexo femenino (10). Es presumible que esta diferencia se deba a la distinta edad promedio de las poblaciones respectivamente consideradas.

Es de amplio conocimiento que el parto antes de los 30 años y la lactancia son considerados como factores protectores en la génesis del CM, la mayor parte de las encuestadas tuvieron hijos antes de los 30 años (144, que representan el $80 \%$ de este subgrupo, y la mayoría (151 pacientes, o sea el 83,8 \% dieron de mamar durante más de 6 meses.

Ciento dieciséis encuestadas $(39,1 \%)$ consumía alcohol, siendo esta cifra algo inferior a la reportada en la Primera Encuesta Nacional de Factores de Riesgo de Enfermedades Crónicas no 
Transmisibles (ECNT), que fue del 63,7\% (10) y la mayoría de las que ingerían alcohol (104, equivalente al $89,6 \%$ ) lo hacían menos de tres veces a la semana.

Si bien entre los factores de riesgo indagados no se encontraba el tabaquismo y múltiples estudios sugieren que existe un riesgo más elevado de desarrollar CM en la población fumadora, la relación entre el tabaquismo y el desarrollo del CM es difícil de analizar, dado que hasta el $50 \%$ de las mujeres fumadores consumen alcohol, un factor de riesgo conocido para el desarrollo de la enfermedad (11).

Por otra parte, la asociación entre un índice de masa corporal alto y el riesgo de desarrollar CM en las mujeres postmenopáusicas también es conocido (12) y tampoco fue indagado en el cuestionario, por lo que queda pendiente la evaluación de estos factores en un futuro.

La mayoría de las mujeres mayores de 50 años realizaban control mamográfico y clínico al menos en forma bianual (117, esto es un $90 \%$ de dicho subgrupo).

Según los datos aportados por la CHLC, durante el año 2014 se realizaron a nivel nacional 95.935 mamografías. Si tenemos en cuenta los datos aportados por el Instituto Nacional de las Mujeres, en nuestro país viven 248.367 mujeres de entre 50 y 64 años. Tomando en consideración que la gran mayoría de exámenes mamográficos se efectúan a mujeres de entre 50 a 70 años, podríamos realizar una extrapolación de los datos, y presumir que más la mitad de las mujeres de entre 50 a 64 años se realiza mamografía en forma bianual. Tal porcentaje, relativamente elevado, traduciría sin duda el impacto de las campañas de prevención realizadas con la CHLC y el Ministerio de Salud Publica

Empero al analizar los resultados conseguidos debemos considerar que la encuesta fue efectuada en el ámbito de las jornadas de prevención de CM; vale decir que la totalidad de las encuestadas asisitió por inquietud en una conferencia de prevención de la enfermedad, lo que acarrea un sesgo muy importante y restringe la posibilidad de extrapolar los resultados a la población general. En este sentido debemos tener en consideración que el $12,1 \%$ de las encuestadas tenían antecedentes personales y el $26 \%$ tenían antecedentes familiares de CM, lo cual podría conllevar un interés de base por parte de las asistentes y eventualmente generar una alteración en el análisis de los resultados.

Igualmente debemos tener en cuenta que la edad media de las encuestadas fue de 36 años (es decir que se trata de una población joven), lo que también podría ser una limitación significativa para extrapolar o proyectar estos resultados en mujeres uruguayas de otras franjas etarias.

Así queda pendiente para estudios venideros la valoración de los factores de riesgo y protectores para el CM en una población más amplia.

\section{CONCLUSIONES}

Si bien, debido a las características de este trabajo, sólo nos es posible alcanzar conclusiones sobre las mujeres incluidas, los datos recogidos nos permiten conocer mejor el perfil epidemiológico de 
la población uruguaya, lo que puede contribuir a las prácticas de prevención. De manera semejante a lo reportado por el PRONACAM, 44,9\% de las encuestadas tuvieron algún factor de riesgo para desarrollar la enfermedad. El porcentaje de encuestadas con antecedentes familiares fue inferior a lo evidenciado a nivel nacional y se halló menor porcentaje de sedentarismo, lo que podría explicarse por la edad de las mujeres encuestadas, con una mediana de 36 años. El porcentaje de consumidoras de alcohol fue inferior a la evidenciada en la primera ECNT, y la mayoría de las encuestadas consumía alcohol menos de tres veces a la semana. La mayoría de las encuestadas de más de 50 años realizaban control mamográfico y clínico al menos en forma bianual. Esto sin duda traduce el impacto de las campañas de prevención realizadas con la CHLC y el Ministerio de Salud Publica. 


\section{BIBLIOGRAFÍA}

1. Ministerio de Salud Pública. División Estadística. Estadísticas de mortalidad. (Fecha de acceso: 19 de abril 2019] Disponible en: http://www.msp.gub.uy/EstVitales/

2. CHLCC. Registro Nacional de Cáncer. Situación epidemiológica del cáncer en el Uruguay. Montevideo, mayo 2019. [citado: 21 de abril de 2020] Disponible

en: https://www.comisioncancer.org.uy/Ocultas/Situacion-Epidemiologica-del-Uruguay-en-relacional-Cancer--Mayo-2019-uc108

3. Comisión Honoraria de Lucha contra el Cancer. Informe Anual. Periodo 2012-2016 (Fecha acceso: 20/04/2020). Disponible en: https://www.comisioncancer.org.uy/Ocultas/Cancer-de-MAMAMujeres--uc77

4. Barrios E.; Garau M. Cáncer: Magnitud del problema en el mundo y en Uruguay, aspectos epidemiológicos. Anales de la Facultad de Medicina 2017, 4, 9-46

5. Fletcher RH, Fletcher SW, Fletcher GS. Clinical epidemiology: The essentials. 5th ed. Philadelphia: Lippincott, 2013. 255 p.

6. Key TJ, Verkasalo PK, Banks E. Epidemiology of breast cancer. Lancet Oncol. 2001;2(3):13340.

7. Nelson HD, Tyne K, Naik A, Bougatsos C, Chan B.K. Humphrey L. Screening for breast cancer: an update for the U.S. Preventive Services Task Force. Ann Intern Med 151 (10): 727-37, W237-42, 2009

8. Ministerio de Salud Pública (Uruguay). Programa Nacional de Detección Oportuna de Cáncer de Mama. Evaluación de sus avances a los 7 años de desarrollo. Montevideo: MSP 1998: 33 pp.

9. Camejo N, Castillo C, Artagaveytia N, Hernandez AL, Schiavone A, Milans S et al. Encuestas sobre prevención del cáncer de mama en una población de mujeres uruguayas. An Facultad Med (Univ Repúb Urug). 2018,5(2):63-74.

10. Ministerio de Salud Pública Dirección General de la Salud, División Epidemiología. 2a Encuesta Nacional de Factores de Riesgo de Enfermedades Crónicas No Transmisibles.

11. Gaudet MM, Gapstur SM, Sun J. Active smoking and breast cancer risk: original cohort data and meta-analysis. J Natl Cancer Inst. 2013 Apr;105(8):515-25. Epub 2013 Feb 28.

12. Eliassen AH, Colditz GA, Rosner B, et al. Adult weight change and risk of postmenopausal breast cancer. JAMA. 2006;296(2):193. 